\title{
PReS-FINAL-1016: Micro vesicles as a magnifying glass; uncovering potential biomarkers in juvenile idiopathic arthritis
}

\author{
G Keustermans", B Prakken, W de Jager \\ From 20th Pediatric Rheumatology European Society (PReS) Congress \\ Ljubljana, Slovenia. 25-29 September 2013
}

\begin{abstract}
Introduction
Juvenile idiopathic arthritis (JIA) is a common chronic inflammatory diseases in childhood. Despite remission as a result of a plethora of treatment techniques, the chronic and relapsing nature of the disease requires continuous treatment which causes adverse side effects. It is important to uncover a biomarker that can efficiently predict patient responses to therapy as well as determine if patients will progress or regress as a result of treatment. Micro vesicles are key messengers containing many immune signaling molecules including cytokines, molecules known to play a major role in JIA.
\end{abstract}

\section{Objectives}

Due to the localized inflammation seen in JIA, we aim to analyze if micro vesicles isolated from patients can provide a source of biomarkers, giving specific information on molecules that can be targeted for treatment and allow the disease state to be monitored.

\section{Methods}

Micro vesicles where isolated from the blood and synovial fluid of patients with various subtypes of JIA. Vesicular protein profiles where then compared using Luminex technology.

\section{Results}

Pilot data showed that whole JIA patient plasma and synovial fluid has an inflammatory phenotype expressing high levels of TNF-R1, S100 A12, CXCL9 and CXCL10.
This phenotype is also seen in exoquick isolated plasma micro vesicles however, when micro vesicles are isolated by ultra-centrifugation, this phenomenon disappears. Ultra-centrifugation isolated vesicles express lower levels of IL-6, MIF, TNF-R1, CXCL9 and S100 A12 when compared to whole plasma and healthy control vesicles. An analysis of exoquick background activity on Luminex MIA technology reveals a high level of interference.

\section{Conclusion}

Preliminary data indicates that micro vesicles isolated from JIA patient plasma by ultracentrifugation have low amounts of inflammatory cytokines. In addition, a more in depth investigation into exoquick activity shows that this product interferes with Luminex MIA technology. As a whole data seems to suggest that micro vesicle cytokine levels from individuals with JIA do not reflect the inflammatory process.

\section{Disclosure of interest}

None declared.

Published: 5 December 2013

doi:10.1186/1546-0096-11-S2-P13

Cite this article as: Keustermans et al:: PReS-FINAL-1016: Micro vesicles as a magnifying glass; uncovering potential biomarkers in juvenile idiopathic arthritis. Pediatric Rheumatology 2013 11(Suppl 2):P13. 